\title{
Optimization of the O'Hara-Rudy Model of Human Ventricular Action Potential With Respect to Electrolyte Concentrations and Rate Dependence
}

\author{
Chiara Bartolucci ${ }^{1}$, Elisa Passini ${ }^{2}$, Stefano Severi ${ }^{1}$ \\ ${ }^{1}$ University of Bologna, Cesena, Italy \\ ${ }^{2}$ University of Oxford, Oxford, United Kingdom
}

\begin{abstract}
The "Comprehensive In vitro Proarrhythmia Assay" (CiPA) initiative proposes a new mechanistic, modelinformed, approach to cardiac safety assessment of new drugs, an approach made possible by a deeper understanding of the ionic currents that play a role in QTc prolongation and in the development of torsades de pointes $(T d P)$.

In defining a new paradigm in the field of cardiac safety the proarrhythmic risk would be primarily assessed using preclinical in vitro and in silico human models. In this scenario the best cellular computer model(s) would have to be selected and if necessary improved.

The aim of this study has been the upgrade of the most updated human ventricular cell model in order to: 1) correct its response to $\left[\mathrm{Ca}^{2+}\right]_{o}$ changes; 2) replicate the steady state action potential duration (APD) rate dependency and 3) the S1S2 APD restitution.

The presented model has been validated against the same experimental data used for the original one, in order to verify its consistency and enforce its integration in the use of in silico models for predicting clinical risk of drug-induced arrhythmias.
\end{abstract}

\section{Introduction}

Computational modeling has proven to be a vital tool in cardiac arrhythmia research, and it is expected to be instrumental in the future pipelines for drug safety assessment. Indeed, the Comprehensive in vitro Proarrhythmia Assay (CiPA) initiative seeks to introduce a new cardiac drug safety testing paradigm that combines in vitro drug effects on multiple ion channels, computational modeling of cardiac currents and AP, and the use of human stem-cell derived cardiomyocytes [1,2].

In the present outlook, it is crucial to choose a model which will preserve an immediate and direct relationship to experimentally derived and verifiable data sets. Nowadays the "gold standard" in the in silico human ventricular cellular electrophysiology is the O'Hara-Rudy model (ORd) [3]. However, since models will always be approximations of the "real world", it is necessary to constantly develop and improve them, depending on the specific question to address. For example, a modified version of the ORd model with a novel formulation for the human ether-à-go-go-related (hERG) ionic current that can reproduce temperature-induced changes in major channel gating processes, and optimised for drug testing, has been recently developed by the US Food and Drug Administration [4].

Another aspect concerns the dependence between APD and $\left[\mathrm{Ca}^{2+}\right]_{0}$ variations, since APD changes are very important in arrhythmia onsets, and should be considered in all clinical contexts where electrolyte modifications occur. Unfortunately, most of the published human AP models have not taken into account the dependence between APD and $\left[\mathrm{Ca}^{2+}\right]_{\mathrm{o}}$ variations, and therefore respond in a non-physiological way, i.e. $\left[\mathrm{Ca}^{2+}\right]_{0}$ increases lengthen APD, and viceversa.

The work of Passini \& Severi [5], which is our starting point, has replaced the original $\mathrm{I}_{\mathrm{CaL}}$ Hodgkin-Huxley description, in the ORd model, by a new Markov model, similar to the one used by Decker-Rudy for canine epicardial cells [6]. In that new model, the APD- $\left[\mathrm{Ca}^{2+}\right]_{\text {o }}$ dependence was improved and corrected without altering model behaviour in control conditions, as similarly done for older models in previous works $[7,8]$.

On the other hand, the steady state action potential duration (APD) rate dependency and the S1S2 APD restitution protocols were not well replicated by that model. For this reason, this work proposes an upgrade of the model presented in [5], which preserves the correct APD- $\left[\mathrm{Ca}^{2+}\right]_{0}$ dependence and is able to fit the experimental data of both rate dependence and restitution protocol. 


\section{Methods}

The O'Hara-Rudy human ventricular AP [3] model was chosen for this study because of the large number of human ventricular experimental data obtained from more than 140 hearts used in its construction and evaluation, and its choice within the CiPA initiative [1].

First of all, the L-Type $\mathrm{Ca}^{2+}$ current has been inherited from Passini \& Severi [5] where a Markov model description was used. With respect to this previous work, we have slightly modified the values of some parameters: rates in the $\mathrm{CDI}\left(\mathrm{Ca}^{2+}\right.$-dependent inactivation) loop versus VDI (voltage-dependent inactivation) loop are less faster than in [5] and one kinetic rate is increased $\left(K_{m, n}\right)$. Moreover, the $\mathrm{n}$ gate kinetics were defined with the algebraic resolution of the differential equations (fast enough to be assumed in steady state) which described the interaction between $\mathrm{Ca}^{2+}$ and Calmodulin $(\mathrm{CaM})$ bound to L-type $\mathrm{Ca}^{2+}$-channels (eq. 1).

$$
\alpha_{n}=\frac{1-n}{\left(1+\frac{K_{m, n}}{\left[C a^{2+}\right]_{s s}}\right)^{4}}
$$

In order to preserve the achieved APD- $\left[\mathrm{Ca}^{2+}\right]_{0}$ dependence without altering the model behaviour in control conditions and to gain a good fitting of the steady state APD rate dependency and the S1S2 APD restitution experimental data, an optimization procedure was implemented to refine the model. In particular, a set of parameters, which are involved in the APD rate dependence and APD- $\left[\mathrm{Ca}^{2+}\right]_{\mathrm{o}}$ dependence, underwent an optimization procedure using the lsqnonlin built-in Matlab function with a cost function taking into account the differences between the experimental and simulated data of the steady state APD rate dependence (for BCL = 400, 1000, $2000 \mathrm{~ms}$ ), the S1S2 APD restitution ( $\mathrm{S} 2=10$, $20,30,40,50,70,100,150,200,500 \mathrm{~ms})$ and a term to control the APD- $\left[\mathrm{Ca}^{2+}\right]_{0}$ dependence.

Since experimental evidence is not clear about $\mathrm{Ca}^{2+}$ movement inside the sarcoplasmic reticulum (SR) [9], the SR was reduced to a single compartment (with a consequent rescaling in the cell geometry), instead of the separation between Network SR (NSR) and Junctional SR (JSR), like some other human models [10,11]. Moreover, the $\mathrm{Ca}^{2+}$ buffering in the SR was reduced.

Since the rate dependence and restitution experimental data, which have been used for validating the model, were obtained with $\left[\mathrm{K}^{+}\right]_{\mathrm{o}}=4 \mathrm{mM}[3]$, we modified the $\left[\mathrm{K}^{+}\right]_{\mathrm{o}}$ concentration accordingly. Moreover, since the liquid junction potential was not compensated when reporting experimental data (as acknowledged by the authors in [3], Comments section), we have shifted the membrane potential dependence of the involved currents ( $\mathrm{I}_{\text {to }}$ activation and inactivation steady state and time constants; $\mathrm{I}_{\mathrm{K} 1}$ rectification, inactivation steady state and time constant; $I_{K s}$ activation steady state and time constants).

Finally we have included in this new model the $\mathrm{I}_{\mathrm{Kr}}$ Markovian formulation from $\mathrm{Li}$ et al. [12] and replaced the SR calcium release equations with a description similar to the one presented in Paci et al. [13].

Other changes involved:

- speed up of $\mathrm{Ca}^{2+}$ diffusion from subsarcolemma space to cytoplasm;

- increase of SERCA pump maximal current;

- increase of $\mathrm{Na}^{+} / \mathrm{Ca}^{2+}$ exchanger maximal current;

- increase of $\mathrm{Na}^{+} / \mathrm{K}^{+}$ATPase maximal current;

- increase of $\mathrm{Ca}^{2+}$ background maximal current;

- reduction of L-type $\mathrm{Ca}^{2+}$ channel permeability;

- increase of late sodium maximal current;

- increase of $\mathrm{I}_{\mathrm{Kr}}$ maximal current;

- reduction of inward rectifier potassium maximal current and increase of the instantaneous rectification slope.

Model differential equations were implemented in Matlab (Mathworks Inc., Natick, MA, USA) and solved with a variable order solver (ode15s), based on numerical differentiation formulas [14]. Simulations were run with the original and modified models at variable $\left[\mathrm{Ca}^{2+}\right]_{0}$ in the range $0.9-2.4 \mathrm{mM}$. Pacing was maintained until steady state AP was reached (1000 s) and $\mathrm{APD}_{\mathrm{X}}$ was measured once membrane voltage reached $\mathrm{X} \%$ of the resting value (as in [3]).

\section{Results}

\subsection{Model Validation}

The present model has been validated against the same experimental data proposed for the original ORd model, and the results were compared with both the corresponding experimental data and the original ORd model.

The $\mathrm{I}_{\mathrm{CaL}}$ Markov model has been validated, in the previous work [5], against the experimental voltageclamp data used for the original one, in order to verify consistency between the two models in control conditions.

The inverse relationship between APD and $\left[\mathrm{Ca}^{2+}\right]_{0}$ was correctly reproduced as shown in Figure 1 (right panel). These simulations were obtained using $\left[\mathrm{K}^{+}\right]_{0}=5.4 \mathrm{mM}$ since this is the commonly accepted physiological value for extracellular potassium. 

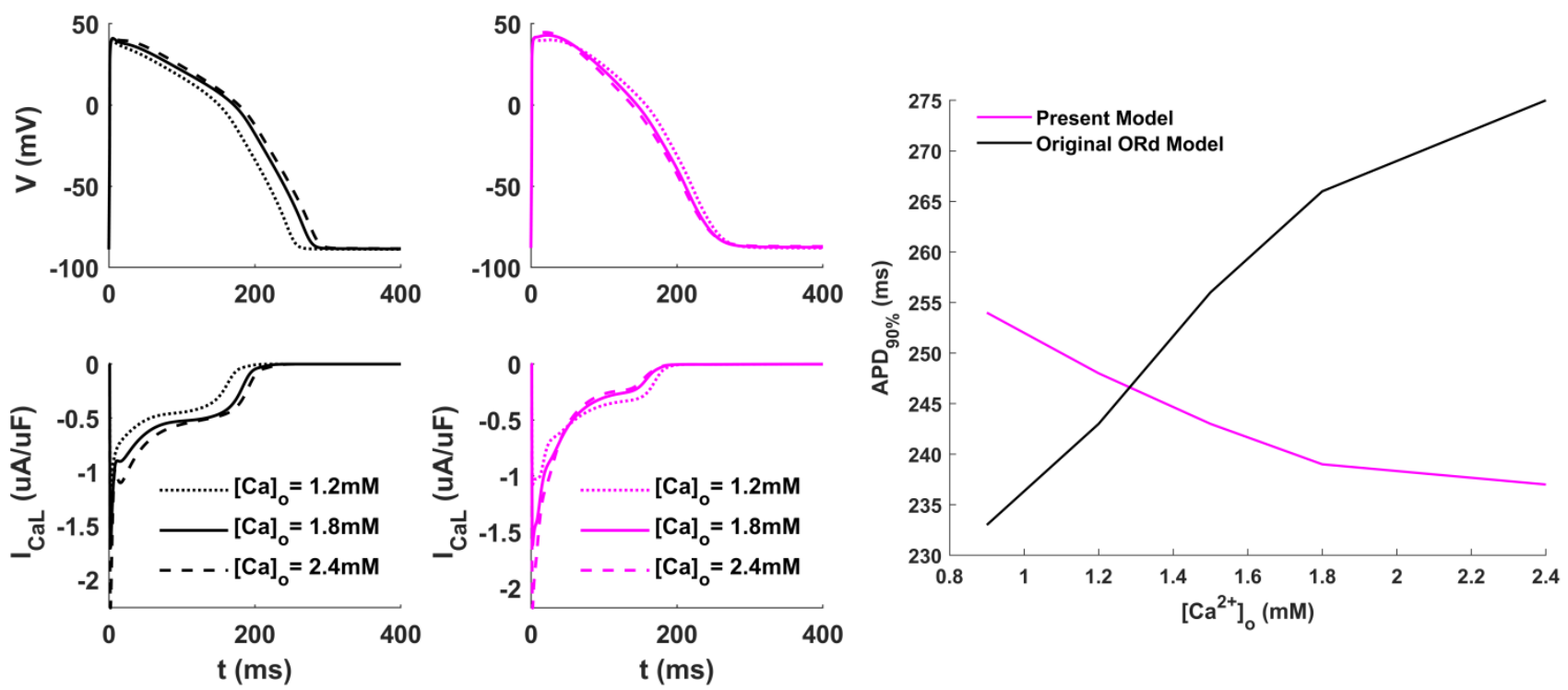

Figure 1. Comparison of the APD- $\left[\mathrm{Ca}^{2+}\right]_{0}$ relationship for the original and the present ORd models. A) Model APs and $\mathrm{I}_{\mathrm{CaLs}}$, using three different $\left.\left[\mathrm{Ca}^{2+}\right]_{0} ; \mathbf{B}\right)$ The APD- $\left[\mathrm{Ca}^{2+}\right]_{0}$ relationship in the range 0.9-2.4mM.

In addition the two protocols of steady state APD rate dependence and S1S2 APD restitution were implemented to replicate the experimental data and compare the model with the original one using $\left[\mathrm{K}^{+}\right]=4 \mathrm{mM}$ also for ORd simulations. The S1S2 APD restitution obtained with the present model is in a good agreement with the experimental one (Figure 2).

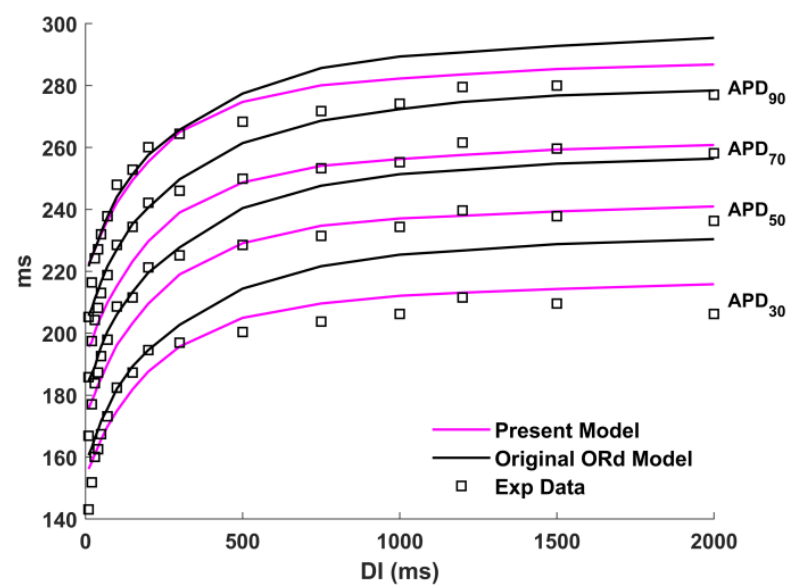

Figure 2. Comparison of the $S_{1} S_{2}$ restitution curves: original ORd model (black lines), present model (pink lines) and experimental data from [3] (squares).
Figure 3 shows the steady state APD rate dependence of the present model compared to the ORd results: in this case for almost all the rates the simulated APD is within the experimental ranges, while the ORd model has longer APDs.

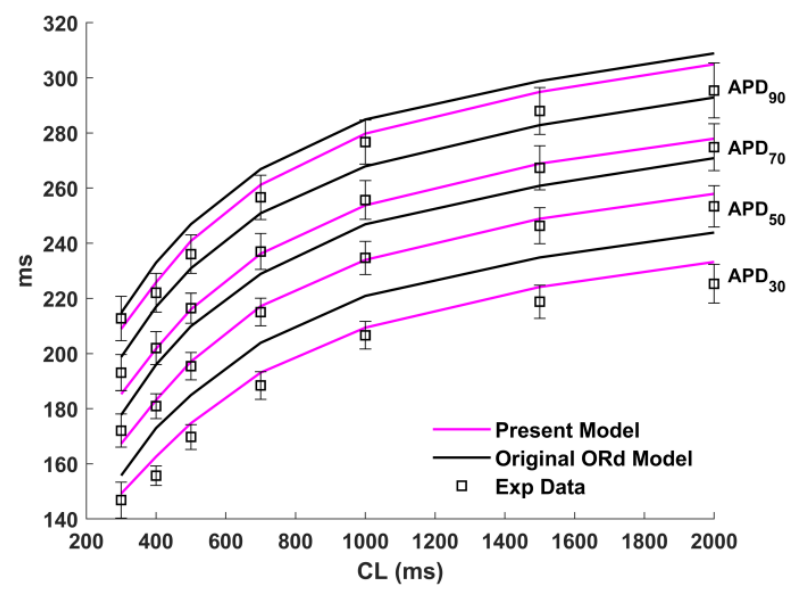

Figure 3. Comparison of steady state APD rate dependence: original ORd model (black lines), present model (pink lines) and experimental data from [3] (squares). 


\section{Discussion and Conclusions}

Starting from a previous L-type $\mathrm{Ca}^{2+}$ current formulation with a Markov model [5], we updated the ORd model in order to reproduce the physiological relation between APD and $\left[\mathrm{Ca}^{2+}\right]_{\mathrm{o}}$ variations and also to reproduce the APD rate dependence and restitution experimental data. The larger sensitivity to CDI of this current model, which is needed in order to reproduce the correct dependence of APD on $\left[\mathrm{Ca}^{2+}\right]_{0}$, could negatively impact on the restitution. Indeed, an eventual decrease of the calcium release at short intervals, due to incomplete JSR refilling, would induce less CDI and APD prolongation instead of the expected APD shortening. For this reason, the SR was reduced to a single compartment. This choice seems reasonable, since the velocity of $\left[\mathrm{Ca}^{2+}\right]_{\mathrm{o}}$ transfer within the various cell compartments is still debated. We also optimised the maximal conductance of other currents, which play a role in these protocols.

When setting $\left[\mathrm{K}^{+}\right]_{\mathrm{o}}=4 \mathrm{mM}$ the experimental data of the S1S2 protocol and of the steady-state rate dependence were very well fitted with all the APD values for almost all the tested rates being within the experimental ranges

Finally, this study highlights the importance of reproducing experimental conditions as closely as possible in the simulations to allow an effective comparison between experiments and simulations for validation.

The next steps will be the verification of the dynamic restitution protocol, relevant in particular for the study of alternans occurrence and the effects of drug block on APD rate dependence, in line with the work of Dutta et al. [15] and for using the new model in the CiPA scenario.

\section{References}

[1] Sager PT, Gintant G, Turner JR, Pettit S, Stockbridge N. Rechanneling the cardiac proarrhythmia safety paradigm: A meeting report from the Cardiac Safety Research Consortium. Am Heart J 2014;167:292-300.

[2] Colatsky T, Fermini B, Gintant G, Pierson JB, Sager P, Sekino Y, et al. The Comprehensive in Vitro Proarrhythmia Assay (CiPA) initiative - Update on progress. J Pharmacol Toxicol Methods 2016;81:1520.

[3] O'Hara T, Virág L, Varró A, Rudy Y. Simulation of the undiseased human cardiac ventricular action potential: Model formulation and experimental validation. PLoS Comput Biol 2011;7.

[4] Li Z, Dutta S, Sheng J, Tran PN, Wu W, Chang K, et al. Improving the in silico assessment of proarrhythmia risk by combining hERG (Human Ether-à-go-goRelated Gene) channel-drug binding kinetics and multichannel pharmacology. Circ Arrhythmia Electrophysiol 2017;10.

[5] Passini, Elisa; Severi S. Computational analysis of extracellular calcium effects on an improved human ventricular action potential model. Comput. Cardiol., Decker KF, Heijman J, Silva JR, Hund TJ, Rudy Y. Properties and ionic mechanisms of action potential adaptation, restitution, and accommodation in canine epicardium. Am J Physiol Heart Circ Physiol 2009;296:H1017-26.

[7] Severi S, Corsi C, Cerbai E. From in vivo plasma composition to in vitro cardiac electrophysiology and in silico virtual heart: the extracellular calcium enigma. Philos Trans A Math Phys Eng Sci 2009;367:2203-23.

[8] Grandi E, Pasqualini FS, Pes C, Corsi C, Zaza A, Severi S. Theoretical investigation of action potential duration dependence on extracellular $\mathrm{Ca} 2+$ in human cardiomyocytes. J Mol Cell Cardiol 2009;46:332-42.

[9] Bers DM, Shannon TR. Calcium movements inside the sarcoplasmic reticulum of cardiac myocytes. J Mol Cell Cardiol 2013;58:59-66.

[10] ten Tusscher KHWJ, Noble D, Noble PJ, Panfilov A V. A model for human ventricular tissue. Am J Physiol Circ Physiol 2004;286:H1573-89.

[11] Grandi E, Pasqualini FS, Bers DM. A novel computational model of the human ventricular action potential and $\mathrm{Ca}$ transient. J Mol Cell Cardiol 2010;48:112-21.

[12] Li Z, Dutta S, Sheng J, Tran PN, Wu W, Colatsky T. A temperature-dependent in silico model of the human ether-à-go-go- related ( hERG ) gene channel. J Pharmacol Toxicol Methods 2016;81:233-9.

[13] Paci M, Pölönen R, Cori D, Penttinen K. Automatic Optimization of an in Silico Model of Human iPSC Derived Cardiomyocytes Recapitulating Calcium Handling Abnormalities. Front Physiol 2018;9:1-14.

[14] Shampine LF, Reichelt MW. The MATLAB ODE Suite. SIAM J Sci Comput 1997;18:1-22.

[15] Dutta S, Chang KC, Beattie KA, Sheng J, Tran PN, Wu WW, et al. Optimization of an in silico cardiac cell model for proarrhythmia risk assessment. Front Physiol 2017;8:1-15.

Address for correspondence:

Stefano Severi

Department of Electrical, Electronic

and Information Engineering,

University of Bologna,

Via Macchiavelli, 47522 Cesena (FC),

Italy

stefano.severi@unibo.it 